\title{
Distribution of Static Deformations along the Friction Lining of the Brake Mechanism in the Cars of GAZelle Series
}

\author{
H.V. Vardanyan, N.A. Bazikyan, V.A. Vardanyan, S.N. Bazikyan \\ Armenian National Agrarian University \\ henrik1993@inbox.ru, vard.v.25@mail.ru, bazikyansusanna@gmail.com
}

\section{ART I C L E I N F O}

Keywords:

brake mechanism,

friction lining,

shoe,

radial displacement,

tangential displacement, wear

\begin{abstract}
A B S T R A C T
The study of the shoe lining in the drum brake mechanism is of paramount importance to evaluate its wear regularity. Thereto, the computational scheme of the drum brake-shoe mechanism has been designed, the calculation formulae of tangential and radial components of the external points displacement in the lining have been analyzed, by means of which their numerical values have been determined, the diagram of the radial displacements has been designed and compared with descriptors of the wear change along the friction lining, according to which they alter with similar regularities.
\end{abstract}

\section{Introduction}

To determine the pressure distribution pattern along the shoe lining and to evaluate its effect on the wear of the brake mechanism elements, it is necessary to study the stress-strain state of the shoe lining. As a rule, the brake shoes have a high bending rigidity and relatively rigid supports, the deformation of which can be ignored. It is evident that the main deformable element of the drum brake-shoe mechanism is the friction lining which has a thickness of $10 \mathrm{~mm}-16 \mathrm{~mm}$ and an elasticity modulus smaller in several ranges as compared to that of the shoe substance. Let's examine the work of the brake mechanism to determine its stress-strain state (Vardanyan, et al., 2019).

Since the inner radius of the brake drum and the outer radius of the shoe are not equal, their contact throughout the whole friction plane is not simultaneous and a friction pair contact with small area appears at the start of braking, which grows up along with the increase of the shoe pressing $P$ power encompassing the full friction area of the lining and drum. To compress the drum, the shoe lining completely rotates round the $O_{1}$ point and all external points of the lining rotate round the radius $\rho_{i}$ (Figure 1) (Bazikyan, Djinyan, 2005).

\section{Materials and methods}

Let's analyze the triangle $O_{1} A O$ introduced in figure 1 to determine the external points $A_{i}$ of the lining, where $O_{1} O$ is the radius $R_{l}$ of the lining circle, $A O_{i}$ is the radius of the drum $R_{d}$ respectively, $R_{d}-\left(R_{l}+h\right)=\Delta$ is the clearance between the drum and lining, and $h$ is the thickness of lining. 


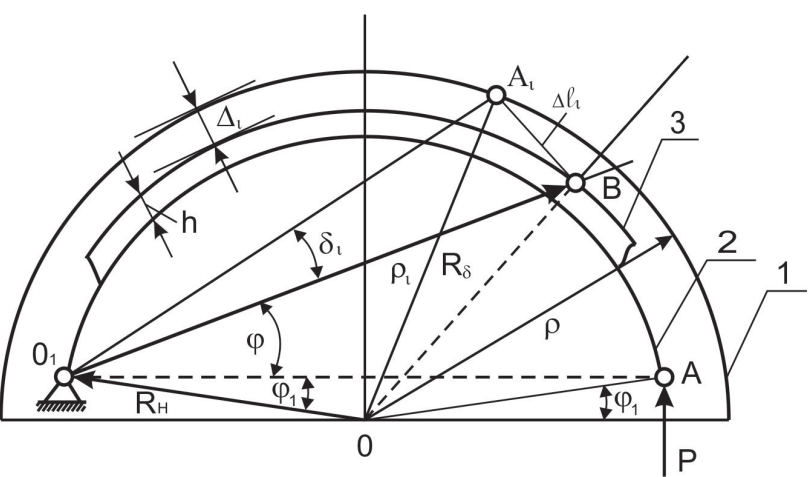

Figure 1. Drum brake-shoe mechanism. 1-drum, 2- shoe, 3- lining (composed by the authors).

Let's determine the current value of the $\rho_{i}$ radius vector depending on the rotation angle $\varphi_{i}$ of the $A_{i}$ point through the $O_{1} A_{i} O$ isosceles triangle considering that $\triangleleft \varphi_{i}=\triangleleft 0_{1} A B$ is a variable value for different $A_{i}$ :

$$
\rho_{i}=2 R_{i} \cdot \cos \left(\varphi+\varphi_{i}\right),
$$

it is accepted that $O_{1} O \approx O B=R_{l}$,

we'll get from triangle $O_{1} A_{i} O$ :

$$
R_{d}=R_{d}^{2}+\rho_{i}^{2}-2 R_{i} \cdot \rho_{i}^{2} \cdot \cos \left(\varphi+\varphi_{i}+\delta_{i}\right)
$$

Placing the equation (1) in the (2) one and making some modification we'll have the following:

$$
\frac{R_{d}^{2}-R_{l}}{4 R_{l}^{2}}=\cos ^{2} \beta\left(1-\cos \delta_{i}\right)+\frac{1}{2} \cos 2 \delta_{i} \cdot \sin \delta_{i},
$$

where $\beta=\varphi+\varphi_{i}$.

From the derived (3) equation the rotation angle $\delta_{i}$ of the radius vector for $A_{i}$ point in the brake mechanism can be determined.

The clearance $\Delta$ between the brake lining and drum is expressed through a rather small value due to which the rotation angle $\delta_{i}$ of the radius vector will be small as well, thus it can be accepted that $\sin \delta_{i}=\operatorname{tg} \delta_{i}=\delta_{i}$ and after some modifications the (3) equation will look like the following:

$$
\delta_{i}^{2} \cos ^{2} \beta+\frac{1}{2} \delta_{i} \cdot \sin ^{2} \beta-a_{i}=0,
$$

where. $a_{i}=\frac{R_{d}^{2}-R_{l}}{4 R_{l}^{2}}$
The solution of the derived equation will be as follows:

$$
\delta_{i 1.2}=\frac{\sin \beta \pm \sqrt{\sin ^{2} \beta+a_{i}}}{\cos \beta}
$$

From the derived solution it follows that the rotation angle $\delta_{i}$ of the $\rho_{i}$ radius vector will be at minimum if the first derivative of the (4) expression is equal to 0 :

$$
\sin \beta=\frac{1}{\sqrt{2+a_{i}}} \text { or } \sin \left(\varphi+\varphi_{i}\right)=\frac{1}{\sqrt{2+a_{i}}}
$$

Therefore, the minimum rotation angle of radius vector will be:

$$
\delta_{i}=\frac{a_{i}}{\sqrt{a_{i}+1}} .
$$

Let's determine the value of $a_{i}$ depending on the clearance between the drum and lining, taking into account that $\Delta_{i}=R_{d}-R_{l}$, since while rotating round the point the $O_{1}$ distance of the $A_{i}$ points in the lining from the $R_{l}$ grows up with the size of $\Delta_{i}$. For that purpose after some modification of the expression we'll have:

$$
\begin{gathered}
a_{i}=\frac{R_{d}^{2}-R_{l}^{2}}{4 R_{l}^{2}}, \\
a_{i} \cong \frac{\Delta_{i}}{2 R_{l}} .
\end{gathered}
$$

To contact the drum, the displacement value of $A_{i}$ any point in the lining will be:

$$
l_{i}=\rho_{i} \cdot \delta_{i}
$$

The first point to press the drum will be the one, the radius vector $\rho_{i}$ of which matches the rotation angle $\varphi$ :

$$
\varphi=\arcsin \frac{1}{\sqrt{2+a_{i}}}-\varphi_{1} .
$$

Therefore, all external points of the lining will be displaced with the size of $\Delta l_{i}$ before pressing the drum:

$$
\Delta l_{i}=\rho_{i} \cdot \delta_{(\varphi=0)^{-}} \rho_{i} \cdot \delta_{i},
$$

where $\Delta l_{i}$ is the displacement of $A_{i}$ point in the lining in case of rotation angle $\varphi=\frac{\pi}{2}-\frac{\delta_{i}}{2}$ of $\rho_{i}$ radius vector.

Let's divide the $\Delta l_{i}$ displacement into tangential $V_{i}$ and radial $W_{i}$ components and we'll have:

$$
\left\{\begin{array}{l}
V_{i}=\Delta l_{i} \cos \left(\varphi_{i}+\varphi-\frac{\delta_{i}}{2}\right) \\
W_{i}=\Delta l_{i} \sin \left(\varphi_{i}+\varphi-\frac{\delta_{i}}{2}\right)
\end{array} .\right.
$$




\section{Results and discussions}

Taking into account that the stress-strain state of the lining, the regularity of the pressure distribution along the lining, as well as the wear of the brake mechanism parts are characterized through the components derived in the system of lining displacement (9), let's determine their numerical values and design the descriptor $W_{i}=f\left(\varphi_{i}\right)$ (Figure 2).

The numbered values identify $a_{i}$ for the following changing ranges: $a_{i}=0.1-0.28$, according to which $\delta_{i_{\text {min }}}=0.02 \mathrm{rad}$. The rotation angle $\varphi_{i}$ of the radius vector is determined through the (5) formula:

$$
\varphi_{i}=\operatorname{arc} \sin \frac{1}{\sqrt{2+a_{i}}}-\varphi,
$$

where $\varphi=10^{\circ}$.

The computation results are introduced in Table.

The derived descriptor (Figure 2) testifies that the radial displacements of the external points in the lining grow up along with the increase of rotation angle of the points and it amounts to the maximum in the frontal part of the lining. Such characteristic will cause to the same regularity in the pressure distribution along the lining and to the similar descriptors of the lining wear (Figure 3), (Vardanyan, 2020).

Table. The computation results of the displacement components*

\begin{tabular}{|c|c|c|c|c|c|c|c|}
\hline$a_{i}$ & $\begin{array}{c}\varphi_{i} \\
\text { radius }\end{array}$ & $\begin{array}{c}\varphi_{i} \\
\text { degree }\end{array}$ & $\begin{array}{c}\rho_{i} \\
\boldsymbol{m} \boldsymbol{m}\end{array}$ & $\stackrel{\delta_{i,}}{\text { radius }}$ & $\begin{array}{c}l_{i} \\
m m\end{array}$ & $\begin{array}{c}\Delta l_{i} \\
m \boldsymbol{m}\end{array}$ & $\begin{array}{c}W_{i} \\
m m\end{array}$ \\
\hline 1.7716 & 0.27911 & 16.0 & 240.02717 & 1.06414 & 255.423 & 15.1723 & 0.134122 \\
\hline 1.56278 & 0.29656 & 17.0 & 237.47569 & 0.97621 & 231.825 & 14.4 & 1.010796 \\
\hline 1.37283 & 0.314 & 18.0 & 234.85195 & 0.89122 & 209.304 & 13.6296 & 1.769448 \\
\hline 1.19958 & 0.33144 & 19.0 & 232.15674 & 0.80883 & 187.776 & 12.8533 & 2.412668 \\
\hline 1.04114 & 0.34889 & 20.0 & 229.39089 & 0.72874 & 167.166 & 12.0624 & 2.941236 \\
\hline 0.8959 & 0.36633 & 21.0 & 226.55523 & 0.65066 & 147.41 & 11.2474 & 3.353951 \\
\hline 0.76247 & 0.38378 & 22.0 & 223.65064 & 0.57433 & 128.449 & 10.3977 & 3.647379 \\
\hline 0.63962 & 0.40122 & 23.0 & 220.67798 & 0.49952 & 110.233 & 9.50074 & 3.815496 \\
\hline 0.52629 & 0.41867 & 24.0 & 217.63818 & 0.426 & 92.7135 & 8.54179 & 3.84916 \\
\hline
\end{tabular}

*Composed by the authors.

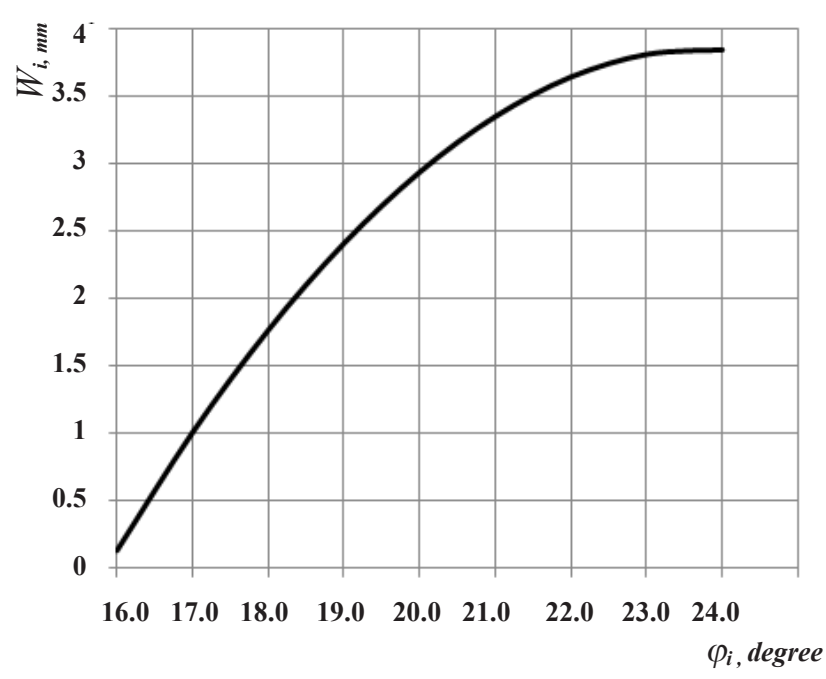

Figure 2. Radial displacement diagram of the external points in the friction lining (composed by the authors).

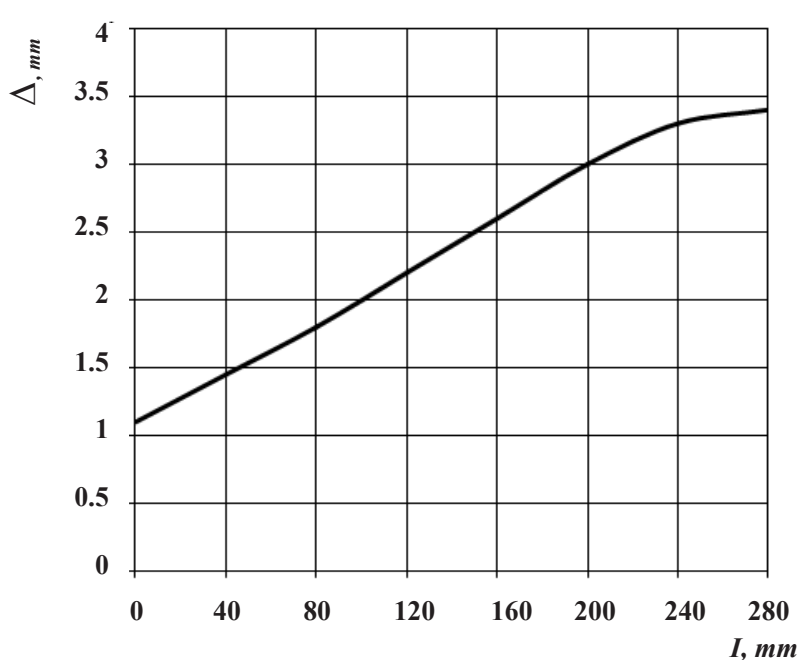

Figure 3. Description of wear change along the friction lining (composed by the authors). 


\section{Conclusion}

Per the descriptor $W_{i}=f\left(\varphi_{i}\right)$ of the radial displacement changes in the external $A_{i}$ points of the lining in the brakeshoe mechanism a conclusion can be drawn that along with the increase of the shoe rotation angle $\varphi_{i}$ the radial displacements grow up with the sine theorem accepting the minimum value at the shoe base equal to $0.13 \mathrm{~mm}$ and the maximum one at the frontal part of the shoe amounting to $3.84 \mathrm{~mm}$. Such characteristics will simultaneously bring forward similar descriptors in the wear changes and pressure distribution along the lining.

\section{References}

1. Bazikyan, N. A., Djinyan, A.M. (2005). Distribution
Nature of Displacement Points in the Shoe during the Braking Process //Bulletin of the Armenian Agricultural Academy, - № 4, - pp.71-74.

2. Vardanyan, H.V. (2020). PhD in Technical Sciences on the Topic of "Evaluating the Reliability of the Brake System in the Minibus of GAZelle Series through the Resource Indicators of the Machine Parts", - Yerevan, - p. 154.

3. Vardanyan, H., Bazikyan, N., Vardanyan, V. (2019). Reliability Assessment of the Brake System in Gazelle Microbus through Resource Indicators of the Limiting Machine Parts. - "Agriscience and Technology", Armenian National Agrarain University. Yerevan, - N 1, - pp. 27-29. 